\title{
Principles for clinical care of patients with COVID-19 on medical units
}

\author{
David W. Frost MD, Rupal Shah MD MHPE, Lindsay Melvin MD MHPE, Miguel Galán de Juana MD, \\ Thomas E. MacMillan MD MSc, Tarek Abdelhalim MD, Alison Lai MD, Shail Rawal MD MPH, \\ Rodrigo B. Cavalcanti MD MSC
}

Cite as: CMAJ 2020 June 29;192:E720-6. doi: 10.1503/cmaj.200855; early-released June 3, 2020

H

ealth systems have responded to the coronavirus disease 2019 (COVID-19) pandemic by prioritizing critical care capacity; however, most patients with COVID-19 are cared for outside intensive care units (ICUs). ${ }^{1,2}$ In many jurisdictions, the first wave of cases is waning, ${ }^{3}$ but as public health measures such as physical distancing are relaxed, subsequent waves seem inevitable. ${ }^{4}$ Protocols are needed to guide care for patients with COVID-19 who are not critically ill, ideally on separate inpatient units designed to optimize patient care while limiting the spread of severe acute respiratory syndrome coronavirus 2 (SARS-CoV-2) from those with the virus to others.

We considered available evidence and drew on our local experiences in Madrid and Toronto to propose an approach to the development of COVID-19 units and care teams. We discuss the layout of COVID-19 units, structure of the physician care team, clinical assessment and rounding protocols, evidencebased methods to foster culture change and ways to mitigate the adverse effects of isolation for patients.

\section{How should COVID-19 units be organized?}

In March 2020, physicians in Spain who were treating patients admitted to hospital with COVID-19 recognized the risk of SARSCoV-2 spreading to other patients and health care workers. ${ }^{5}$ To mitigate this risk and drawing on experiences shared by physicians in Wuhan, China, they created COVID-only units, which delineated zones by risk of contamination using floor markings and signage, restructured medical teams and optimized use of personal protective equipment (PPE). ${ }^{6}$ Our group at University Health Network in Toronto adapted these principles to our context.

Treating infected patients on COVID-19 units mitigates the spread of SARS-CoV-2 to patients who are not infected. Cohorting also enables changes to a unit's layout to limit the risk of nosocomial outbreaks and infection of health care workers. Concentric zones of risk are established around individual patient rooms (red zone, highest risk) to the hallway (green zone, intermediate risk) and to the nursing station (blue zone, lower risk) (Figure $1 \mathrm{~A}-\mathrm{C}$ ). Principles include minimizing traffic between zones and providing visual barriers to prompt use of full PPE in the red zone, with proper doffing of PPE and hand hygiene before exiting. Because

\section{KEY POINTS}

- Hospitals must develop comprehensive plans to care for patients with coronavirus disease 2019 (COVID-19) on medical units, to ensure strict infection control and staff safety.

- Division of medical units into clear risk zones, buddy systems to facilitate spotting of personal protective equipment donning and doffing procedures, and standardization of care protocols may decrease the risk of nosocomial outbreaks of COVID-19 and infection of health care workers.

- When considering how to structure medical care teams, it is important to consider how rapidly teams can be scaled to need, their ability to integrate redeployed physicians and ensure team members' safety, the long-term sustainability of the team, and whether it is possible to maintain a degree of continuity of care.

- Measures aimed at early detection of clinical deterioration and rapid transfer of patients with COVID-19 to the intensive care unit should be instituted.

- Care teams should be aware of the potential adverse effects of isolation for patients and adopt patient-centred practices aimed at mitigating these effects despite restrictions related to infection control.

many health care workers interact with potential fomites in the nursing station (e.g., charts, keyboards and telephones), maintaining nursing stations as separate decontaminated zones is critical.

Effective cohorting of patients requires readily available and reliable testing for SARS-CoV-2, with rapid return of results. Current limitations in the sensitivity of screening tests for SARS-CoV-2 infection necessitate the use of clinical judgment to guide repeat testing to identify patients whose care should be delivered on a COVID-19 unit. $^{7}$

\section{What is the best approach to constructing a COVID-19 medical team?}

When deciding on the composition of the COVID-19 medical team, it is important to consider how rapidly teams can be scaled to need, their ability to integrate redeployed physicians and ensure team members' safety, the long-term sustainability of the team, and whether it is possible to maintain a degree of 
continuity of care. Where possible, in assigning clinicians, it is prudent to consider their risk factors for severe illness, such as their age, immune status and underlying medical conditions, should they become infected. ${ }^{8,9}$

A standard COVID-19 medical team should be easily replicable, given the imperative to respond to rapid increases in admissions. Large surges in illness may necessitate redeployment of nonhospitalists, as occurred during local outbreaks of novel influenza $\mathrm{A}(\mathrm{H} 1 \mathrm{~N} 1)$ and SARS, for example. ${ }^{10,11}$ Integration of redeployed physicians also leverages the expertise of relevant specialties in the care of patients with COVID-19 (e.g., respirology, infectious diseases and palliative care). A structure comprising an experienced general internist or hospitalist and another clinician, who may be a senior resident, a redeployed physician with relevant skills or a nurse practitioner, may help to achieve scalability and replicability goals.

A 2-clinician "buddy system" - previously described as part of pandemic planning for $\mathrm{H} 1 \mathrm{~N} 1$ in a letter to the editor of $C M A J^{12}-$

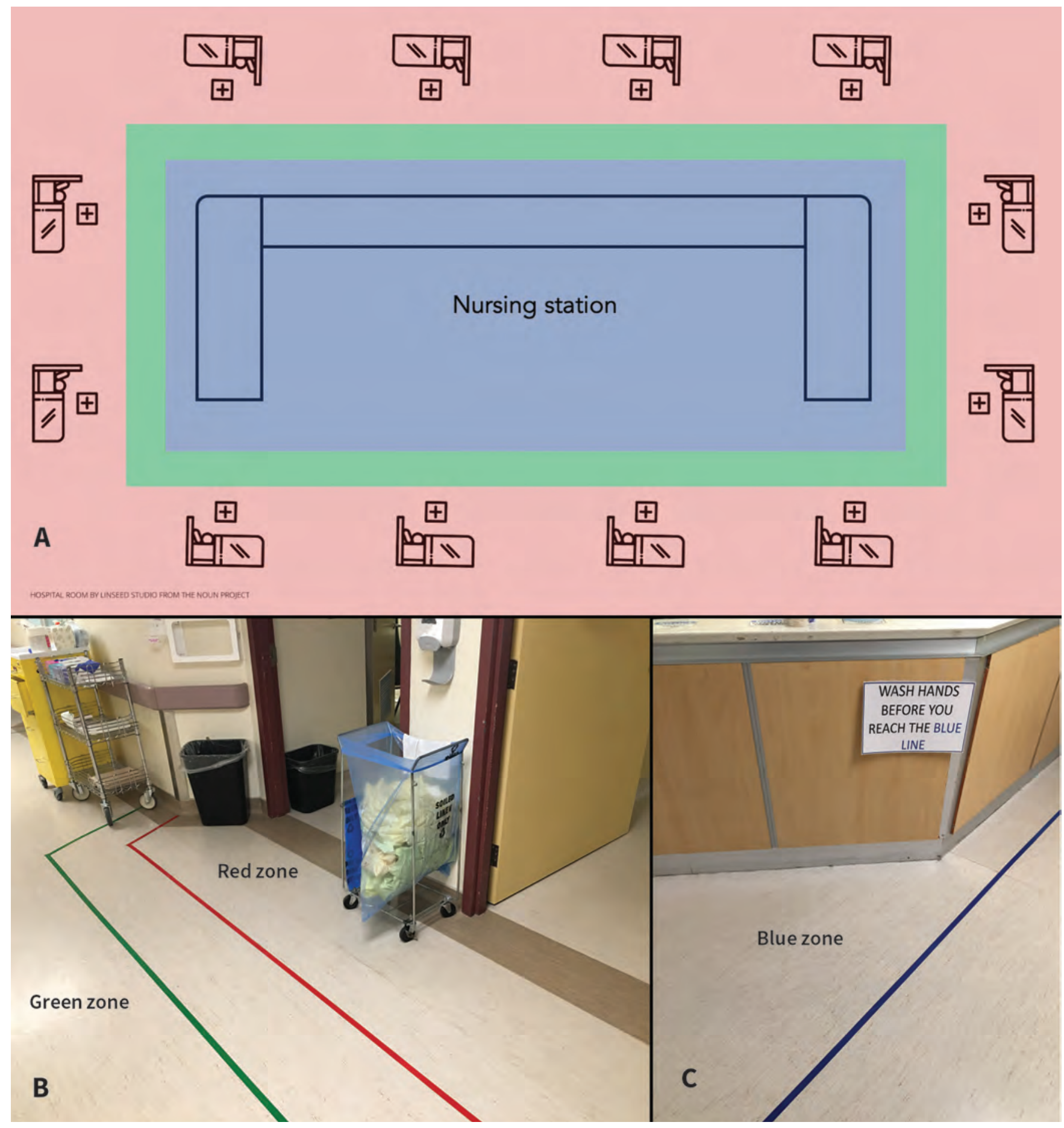

Figure 1: (A) Layout of the coronavirus disease 2019 (COVID-19) unit by zone. (B) Red and green zones and (C) blue zone. 
allows separation of tasks, with attendant advantages for infection control. For example, clinician 1 may perform all physical examinations of patients and enter only red zones (with careful donning and doffing of PPE), whereas clinician 2 would stay in the green zone, serve as a PPE spotter and perform other tasks (e.g., documentation, checking results, and ordering medications and tests), and would be available to offer or call for help. In this system, clinicians entering patient rooms are less likely to contaminate the environment. The structure also encourages clinical discussion and collaborative decision-making. In our experience, alternating roles between clinician 1 and 2 every 3-4 days minimizes fatigue and balances risk exposure.

Other considerations include careful scheduling to allow clinicians sufficient rest after service on COVID-19 wards - which can be stressful - and using occupational health guidelines for testing clinicians for SARS-COV-2.

\section{How should ward rounds be conducted?}

\section{Standard procedures and checklists}

The formalization of procedures for conducting rounds on wards and their dissemination among providers of care for those with COVID-19 allows consistency of process and facilitates onboarding of redeployed physicians and new team members.

Priorities for ward rounds should be optimal patient care, safety of health care workers and meticulous infection control. In our system, ward rounds are conducted by the 2 clinicians and may include the unit charge nurse and interprofessional care team members. Modelled after surgical time outs, rounds begin and end with a safety checklist completed in the green zone (Figure 2). ${ }^{13}$ Rounds are designed to maximize efficiency in providing patient care while eliminating traffic to and from the nursing station (protecting the blue zone), minimizing unnecessary PPE use and maintaining separation among all zones. The use of a mobile workstation and templated daily progress notes during ward rounds allows for electronic notes to be typed and printed remotely, minimizing contact between clinicians in the red zone and the paper chart.

\section{Personal protective equipment}

Given the concerns with supply chains for PPE, ${ }^{14}$ teams must be aware of the specific equipment recommended by local infection prevention and control departments. Careful PPE donning and doffing procedures should be observed to reduce the risk of staff acquiring infection. Similar to experiences in other settings, our teams found that donning and doffing is best performed under close observation by a PPE spotter, ${ }^{15}$ who can be any member of the health care team who observes the entire procedure from the green zone. The spotter must be empowered to intervene as required. We take a deliberate pause during donning and doffing to allow focused attention on these critical tasks.

\section{Physical examination}

For most patients on COVID-19 units, a critical component of daily assessments is the identification of those requiring urgent ventilatory support. Therefore, clinical assessments should focus on signs of respiratory distress and early signs of acute respiratory distress syndrome (ARDS). Careful clinical observation can help identify signs of respiratory distress without need for auscultation. For example, the presence of gasping has a positive likelihood ratio $(\mathrm{LR}+)$ of 7.8 for severe respiratory distress. ${ }^{16}$ The presence of any 3 signs of serious respiratory dysfunction (e.g., suprasternal retraction, nasal flaring or Hoover sign) has an LR+ for severe respiratory distress of 18.9. All these signs can be ascertained without the need for auscultation of the chest.

Identification of early manifestations of ARDS is another priority for which auscultation has limited use. In a 2004 study that compared diagnostic performances of auscultation, chest radiography and lung ultrasonography with thoracic computed tomography (CT), auscultation had poor sensitivity (34\%) and low diagnostic accuracy (55\%, compared with CT of the thorax) for alveolar interstitial syndrome. ${ }^{17}$

The marginal added value of auscultation above careful clinical observation must be balanced with the increased risk of nosocomial infection conferred by the stethoscope. ${ }^{18}$ Viable SARS-CoV-2 has been detected for up to 72 hours on plastic and stainless steel surfaces. ${ }^{14}$ Therefore, the routine use of stethoscopes for patients with COVID-19 should be limited, and, in clinical situations where auscultation is deemed useful (e.g., assessment for wheezing in patients with suspected bronchoconstriction), extra attention to infection control should be exercised. The potential value of other physical examination manoeuvres should be weighed in a similar way: for example, we found that physical examination for deep venous thrombosis in patients with elevated levels of D-dimers helped to guide clinical decision-making.

\section{Computed tomography and point-of-care ultrasonography}

Although CT is useful as an ancillary test for diagnosing COVID-19, transferring patients who are positive for SARS-CoV-2 to scanner locations poses a risk of infection to staff. ${ }^{7}$ This has raised interest in point-of-care ultrasonography (POCUS) of the chest, which can help differentiate causes of acute respiratory failure. ${ }^{17}$ However, ultrasonography findings reported for patients with COVID-19 (i.e., pleural thickening and patchy B-lines) are nonspecific. ${ }^{19}$ In addition, the ultrasonography devices require thorough decontamination between patients, which can interfere with workflow on routine ward rounds. The need for operator expertise is another barrier to use of POCUS. ${ }^{20}$

\section{Early warning systems for clinical deterioration}

The respiratory status of patients with COVID-19 who are admitted to hospital may deteriorate rapidly, with progression from minimal oxygen requirements to respiratory failure in hours. ${ }^{21}$ Early detection of deterioration may allow definitive airway management to be performed in a controlled rather than an emergency setting, which is preferred as emergent intubation may increase the risk of infection among health care workers..$^{22}$ Moreover, early detection of hypoxia can allow for implementation of therapies such as high-flow oxygen and prone positioning. However, on medical units, the frequency of monitoring vital signs is typically limited to 4-hour intervals. Increasing the frequency of in-person monitoring increases risk of exposure to the virus. 


\section{COVID-19 \\ PRE-ROUNDING CHECKLIST}

\section{Before you begin}

1 ACQUIRE PPE FOR BOTH HCWS

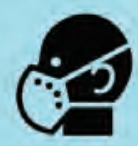

2 OBTAIN AND CLEAN REQUIRED DEVICES

3 DESIGNATE HCW-1 AND
HCW-2
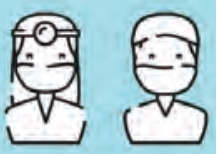

4 HCW-1 SAFETY STATEMENT:

"PLEASE SPOT ME WHEN I'M DONNING AND DOFFING MY PPE. DO NOT HESITATE TO CORRECT THINGS FOR MY SAFETY AND THE SAFETY OF OUR TEAM."

5 PRINT PATIENT LIST

6 LOAD PROGRESS NOTE TEMPLATE ON COMPUTER-ON-WHEELS (COW)

7 ROUNDING SUPPLIES

(PAPER NOTES, DOCTOR'S ORDERS, PENS)

8 ADVISE NURSING AND ALLIED HEALTH TEAM ROUNDING IS TO BEGIN

9 REVIEW OVERNIGHT AND WARD ISSUES

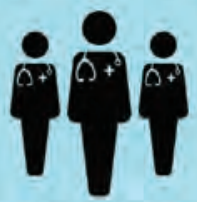

10 REVIEW DISCHARGES AND PREPARE DOCUMENTATION PRIOR TO ROUNDS

MASK BYLUIS PSADOFROMTHE NOUN PROHECT

DOCTORS BY WhISON JOSSPMEROM ThE NOUN

CONTETCALA TORONTOCONDCOUECTIVE WY:L MELVIN, R.SHAH, SRAWA
INFOGRAPHIC RY ALISONLI

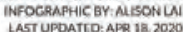

\section{COVID-19 \\ POST-ROUNDING CHECKLIST}

\section{After you've finished}

1 ENSURE ALL PATIENTS HAVE BEEN ROUNDED ON

EVERY PATIENT HAS PROGRESS NOIE AND ORDERS

2 REVIEW NEED FOR

PATIENT RE-ASSESSMENT

LATER IN THE DAY

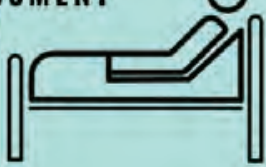

3 PRINT AND/OR ENTER NOTES INTO CHART

4 REVIEW PLAN WITH NURSING AND ALLIED HEALTH REPRESENTATIVES

5 REVIEW OUTSTANDING 'TO DO' TASKS WITH EACH TEAM MEMBER

(MD, RN, PT, OT, SW, SLP. DIETICIAN, ETC.)

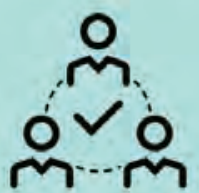

6 DISINFECT AND CLEAN ALL DEVICES

1 POST-ROUND DEBRIEF

- WHAT WORKED WELL

- WHAT NEEDS ADIUSTMENT

- IPAC CONSIDERATIONS

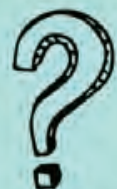

8 UPDATE PATIENTS' LOVED ONES

Figure 2: Infographic outlining the procedures for before and after ward rounds in the coronavirus disease 2019 (COVID-19) unit. Note: HCW = health care worker, IPAC = Infection Prevention and Control, MD = physician, OT = occupational therapist, PPE = personal protective equipment, $\mathrm{PT}=$ physiotherapist, $\mathrm{RN}=$ registered nurse, $\mathrm{SLP}=$ speech language pathologist, $\mathrm{SW}=$ social worker.

To overcome these challenges, we developed a remote pulse oximetry monitoring system, which can be implemented for patients at risk of deterioration and those requiring supplemental oxygen. ${ }^{9}$ Patients who are able to follow directions are provided pulse oximeters with instructions on use and are directed to self-monitor every 1-2 hours and report to nursing staff, without the need for room entry. For patients unable to self-report, we use continuous monitors with audible alerts that combine oximetry and respiratory rate monitoring (Root/Radius-7, Masimo Canada). Early monitoring systems support timely involvement of critical care response teams and rapid transfer to ICU to optimize outcomes. ${ }^{23}$ 


\section{CARING FOR THE PERSON WITH COVID-19}

\section{TIPS FOR PROVIDING PATIENT-CENTRED CARE DURING THE COVID-19 PANDEMIC}
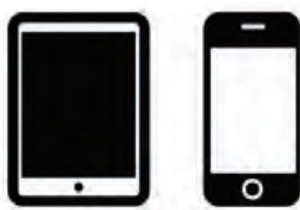

ENCOURAGE USE OF TABLET/CELL PHONE TO REMAIN IN CONTACT WITH LOVED ONES.

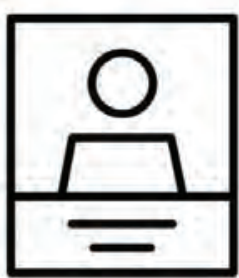

INTRODUCE HEALTHCARE TEAM USING PHOTOS.

PROVIDE PATIENT WITH TEAM INTRODUCTION HANDOUT.

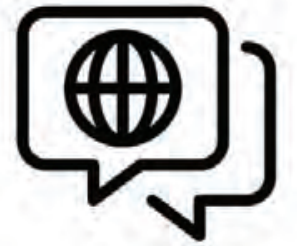

COMMUNICATE USING PATIENT'S PREFERRED LANGUAGE OR INTERPRETER IF POSSIBLE.

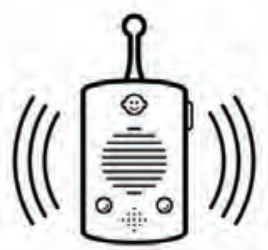

USE BABY MONITORS TO COMMUNICATE WITH PATIENTS IN COVID ISOLATION ROOMS.

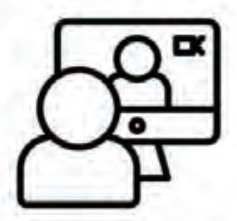

PROVIDE OPPORTUNITY FOR COMMUNICATION WITH LOVED ONES PRIOR TO PROCEDURES OR ESCALATION OF CARE.

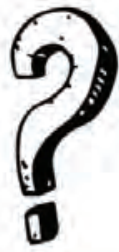

ALLOW TIME TO ADDRESS PATIENT'S QUESTIONS CONCERNS, FEARS. PROVIDE PATIENT WITH COMMUNICATION SHEET.

Figure 3: Tips for maintaining patient-centredness on the coronavirus disease 2019 (COVID-19) unit. 


\section{Fostering a culture of safety}

Instituting large-scale change in clinical practice requires complementary change in workplace culture. Successful measures of infection control require engagement of all stakeholders involved in caring for patients including residents, nurses, porters, environmental service custodians and many others. Culture change is challenging, in particular when practices must be altered rapidly. ${ }^{24}$ Collaboration and clear communication among all stakeholders is essential, because of frequent revisions to care processes that are mandated by changes in the pandemic.

\section{How can the adverse effects of isolation be mitigated for patients?}

Although the long-term experiences of patients with COVID-19 have not yet been described, survivors of ARDS have reported reductions in health-related quality of life up to 5 years after discharge, ${ }^{25}$ and survivors of SARS describe significant sequelae related to in-hospital isolation, home quarantine and stigma. ${ }^{26}$ Patients with COVID-19 are placed under isolation, and many hospitals have enacted no-visitor policies. Strategies to enhance patient-centred care on COVID-19 units where patients are isolated include ensuring dedicated time to address patients' concerns and update families; use of phones and tablets to facilitate communication with loved ones; providing printed photographs of the care team without their PPE for patients; and using medical interpreters when needed (Figure 3).

\section{Conclusion}

The COVID-19 pandemic has affected cities and regions with varied intensity at different times. Practitioners from hard-hit locations have generously shared lessons to the benefit of colleagues in other parts of the world. Much of our workflow was inspired by a widely shared post on social media by one of our authors (M.G., an internal medicine resident at University Hospital Fundación Alcorcón in Madrid, Spain). ${ }^{4}$ We rapidly disseminated our protocols to other units at the University Health Network in Toronto and have shared our approach on an open-access website (www. torontocovidcollective.com). The ability to rapidly disseminate information, iterate protocols and collaborate with physicians around the world will continue to be important through subsequent waves of the pandemic.

The care of patients admitted to hospital with COVID-19 cannot be construed as falling within usual hospital operating procedures. Meticulous planning is required. There are unique challenges regarding necessarily strict infection control procedures, provision of care to potentially large numbers of patients and clinical considerations specific to COVID-19. Based on the existing literature and rapid integration of lessons learned internationally, often via social media, hospitals and care teams can prepare to provide safe and effective patient-centred care in the face of the formidable challenge posed by COVID-19.

\section{References}

1. Grasselli G, Pesenti A, Cecconi M. Critical care utilization for the COVID-19 outbreak in Lombardy, Italy: early experience and forecast during an emergency response. JAMA 2020;323:1545-6.

2. Qiu $\mathrm{H}$, Tong $\mathrm{Z}$, Ma P, et al. Intensive care during the coronavirus epidemic. Intensive Care Med 2020;46:576-8.

3. Maps \& trends 2020. Baltimore: Johns Hopkins University Coronavirus Resource Center; 2020. Available: https://coronavirus.jhu.edu/data (accessed 2020 May 20).

4. Leung K, Wu JT, Liu D, et al. First-wave COVID-19 transmissibility and severity in China outside Hubei after control measures, and second-wave scenario planning: a modelling impact assessment. Lancet 2020;395:1382-93.

5. Wu F, Zhao S, Yu B, et al. A new coronavirus associated with human respiratory disease in China. Nature 2020;579:265-9.

6. @mgalandejuana Galan de Juana M. “We've been in full \#COVID19 mode in my hospital in Alcorcón (Madrid), Spain for the past 2 weeks. This crisis has forced us to adapt like we never thought possible. Here's how we're doing that on our units, in hope that it'll help others across the globe do the same \#FOAMcovid." Twitter 2020 Mar. 22. Available: https://twitter.com/mgalandejuana/status/ 1241764210788442113 (accessed 2020 Apr. 28).

7. Cheng MP, Papenburg J, Desjardins M, et al. Diagnostic testing for severe acute respiratory syndrome-related coronavirus-2: a narrative review. Ann Intern Med 2020 Apr. 13 [Epub ahead of print];M20-1301. doi: 10.7326/M20-1301.

8. Ruan Q, Yang K, Wang W, et al. Correction to: Clinical predictors of mortality due to COVID-19 based on an analysis of data of 150 patients from Wuhan, China. Intensive Care Med 2020 Apr. 6 [Epub ahead of print]:1-4. doi: 10.1007/ s00134-020-06028-z.

9. Zhou F, Yu T, Du R, et al. Clinical course and risk factors for mortality of adult inpatients with COVID-19 in Wuhan, China: a retrospective cohort study. Lancet 2020;395:1054-62.

10. Nickell LA, Crighton EJ, Tracy CS, et al. Psychosocial effects of SARS on hospital staff: survey of a large tertiary care institution. CMAJ 2004;170:793-8.

11. Scarfone RJ, Coffin S, Fieldston ES, et al. Hospital-based pandemic influenza preparedness and response: strategies to increase surge capacity. Pediatr Emerg Care 2011;27:565-72.

12. Ungar T. Pandemic flu buddy system [letter]. CMAJ 2009;181:826.

13. WHO guidelines for safe surgery 2009: safe surgery saves lives. Geneva: World Health Organization; 2009.

14. Rowan NJ, Laffey JG. Challenges and solutions for addressing critical shortage of supply chain for personal and protective equipment (PPE) arising from Coronavirus disease (COVID19) pandemic - case study from the Republic of Ireland. Sci Total Environ 2020 Apr. 6 [Epub ahead of print];725:138532. doi: 10.1016/j.scitotenv.2020.138532.

15. Lockhart SL, Duggan LV, Wax RS, et al. Personal protective equipment (PPE) for both anesthesiologists and other airway managers: principles and practice during the COVID-19 pandemic. Can J Anaesth 2020 Apr. 23 [Epub ahead of print];1-11. doi: 10.1007/s12630-020-01673-w.

16. Tulaimat A, Patel A, Wisniewski M, et al. The validity and reliability of the clinical assessment of increased work of breathing in acutely ill patients. $J$ Crit Care 2016;34:111-5.

17. Lichtenstein D, Goldstein I, Mourgeon E, et al. Comparative diagnostic performances of auscultation, chest radiography, and lung ultrasonography in acute respiratory distress syndrome. Anesthesiology 2004;100:9-15.

18. Maki DG. Stethoscopes and health care-associated infection. Mayo Clin Proc 2014;89:277-80.

19. Thomas A, Haljan G, Mitra A. Lung ultrasound findings in a 64-year-old woman with COVID-19. CMAJ 2020;192:E399.

20. Ma IW, Somayaji R, Rennert-May E, et al. Canadian Internal Medicine Ultrasound (CIMUS) recommendations regarding internal medicine point-of-care ultrasound (POCUS) use during Coronavirus (COVID-19) pandemic. Can J Gen Int Med 2020;15:8-11. 
21. Goh KJ, Choong MC, Cheong EH, et al. Rapid progression to acute respiratory distress syndrome: review of current understanding of critical illness from COVID-19 infection. Ann Acad Med Singapore 2020;49:108-18.

22. Arulkumaran N, McLaren CS, Arulkumaran K, et al. An analysis of emergency tracheal intubations in critically ill patients by critical care trainees. J Intensive Care Soc 2018;19:180-7.

23. Wax RS, Christian MD. Practical recommendations for critical care and anesthesiology teams caring for novel coronavirus (2019-nCoV) patients. Can J Anaesth 2020;67:568-76.
24. Houghton $\mathrm{C}$, Meskell $\mathrm{P}$, Delaney $\mathrm{H}$, et al. Barriers and facilitators to healthcare workers' adherence with infection prevention and control (IPC) guidelines for respiratory infectious diseases: a rapid qualitative evidence synthesis. Cochrane Database Syst Rev 2020;(4):CD013582.

25. Herridge MS, Tansey CM, Matte A, et al. Functional disability 5 years after acute respiratory distress syndrome. N Engl J Med 2011;364:1293-304.

26. Tansey CM, Louie M, Loeb M, et al. One-year outcomes and health care utilization in survivors of severe acute respiratory syndrome. Arch Intern Med 2007;167:1312-20.

\section{Competing interests: None declared.}

This article has been peer reviewed.

Affiliations: Department of Medicine (Abdelhalim, Cavalcanti, Frost, Lai, MacMillan, Melvin, Rawal, Shah), University of Toronto; Division of General Internal Medicine (Abdelhalim, Cavalcanti, Frost, MacMillan, Melvin, Rawal, Shah), University Health Network; HoPingKong Centre for Excellence in Education and Practice, Centre for Innovation in Complex Care (Abdelhalim, Cavalcanti, Frost, Lai, MacMillan, Melvin, Rawal, Shah), University Health Network, Toronto, Ont.; Department of Internal Medicine (Galán de Juana), Hospital Universitario Fundación Alcorcón, Madrid, Spain
Contributors: All of the authors made substantial contributions to the conception and design of the paper, collectively drafted and revised it critically for important intellectual content, gave final approval of the version to be published and agreed to be accountable for all aspects of the work.

Acknowledgement: The authors wish to thank Janet Pilgrim and Kevin White (nurse managers at University Health Network) for their leadership in implementing many of the principles described herein at our hospital.

Correspondence to: David Frost, david.frost@uhn.ca 